\title{
TWO NEW SPECIES OF ERIOCAULON (ERIOCAULACEAE) FROM INDIA
}

\author{
S. Nampr ${ }^{1}$, K. M. Manudev ${ }^{1} \&$ A. K. Pradeep ${ }^{2}$ \\ Two new species of Eriocaulon L. (Eriocaulaceae) from India, E. malabaricum Pradeep \& \\ Nampy and E. pykarense Nampy \& Manudev, are described, illustrated and compared \\ with E. sollyanum Royle and E. ansarii Pradeep \& Sunil, respectively.
}

Keywords. Eriocaulaceae, Eriocaulon malabaricum, Eriocaulon pykarense, India, new species.

\section{INTRODUCTION}

The genus Eriocaulon L. (Eriocaulaceae) with about 400 species (Mabberley, 2008 ) is distributed in tropical and subtropical regions of the world. Ansari \& Balakrishnan (2009) reported 85 species from India. In addition two more species have been described from peninsular India, namely Eriocaulon epedunculatum Potdar et al. and E. baramaticum Shimpale et al. (Yadav et al., 2008; Shimpale et al., 2009). During a recent scientific survey in different parts of southern India, material of this genus was collected by the authors that on critical examination was revealed to be new species. These are described and illustrated here.

\section{SPECIES DESCRIPTIONS}

Eriocaulon malabaricum Pradeep \& Nampy, sp. nov. Figs 1, 2A-B.

Eriocauloni sollyano Royle affinis, a quo imprimis differt receptaculo cylindrico, petalis masculinis glabris, sepalis femineis dimorphis, seminum appendiculis 1-3 e parietibus transversalibus radialibus. - Type: India, Kerala, Wayanad District, Sulthan Bathery, Kuppadi hills, 920 m, on marshy soil, 9 i 2010, Sheba M. Joseph 2729 (holo CALI; iso DEV, E).

Acaulescent herbs. Rootstock up to $1.5 \mathrm{~cm}$. Leaves few, linear, c. $4 \times 0.2 \mathrm{~cm}$, glabrous. Peduncles many, up to $16 \mathrm{~cm}$ long, glabrous, striate; sheath up to $2.5 \mathrm{~cm}$ long, glabrous; limb entire, ovate or rounded at apex. Heads ovate or hemispherical, 3-4 4 2-3 mm. Receptacles cylindric, pilose. Involucral bracts obovate, c. $2.15 \times 0.8 \mathrm{~mm}$, glabrous, acuminate at apex. Floral bracts obovate, c. $1.8 \times 1.5 \mathrm{~mm}$, distally crenate to entire,

\footnotetext{
${ }^{1}$ Department of Botany, St Joseph's College, Kozhikode 673 008, Kerala, India. E-mail for correspondence: santhoshnampy@yahoo.com

2 Calicut University Herbarium, Department of Botany, University of Calicut, Kerala 673 635, India.
} 

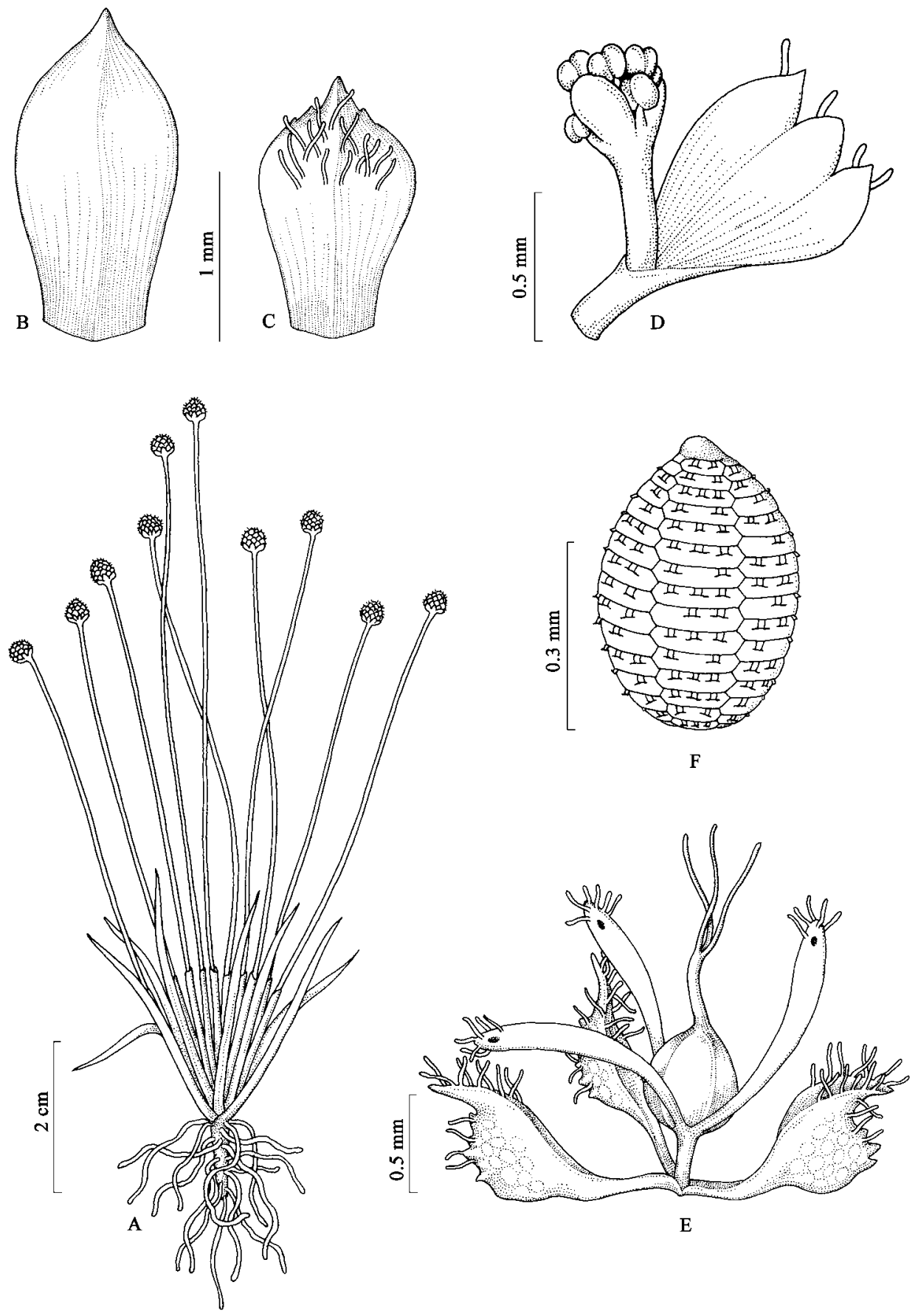

FIg. 1. Eriocaulon malabaricum Pradeep \& Nampy. A, habit; B, involucral bract; C, floral bract; D, male flower; E, female flower; F, seed. Drawn by Manudev from the holotype. 

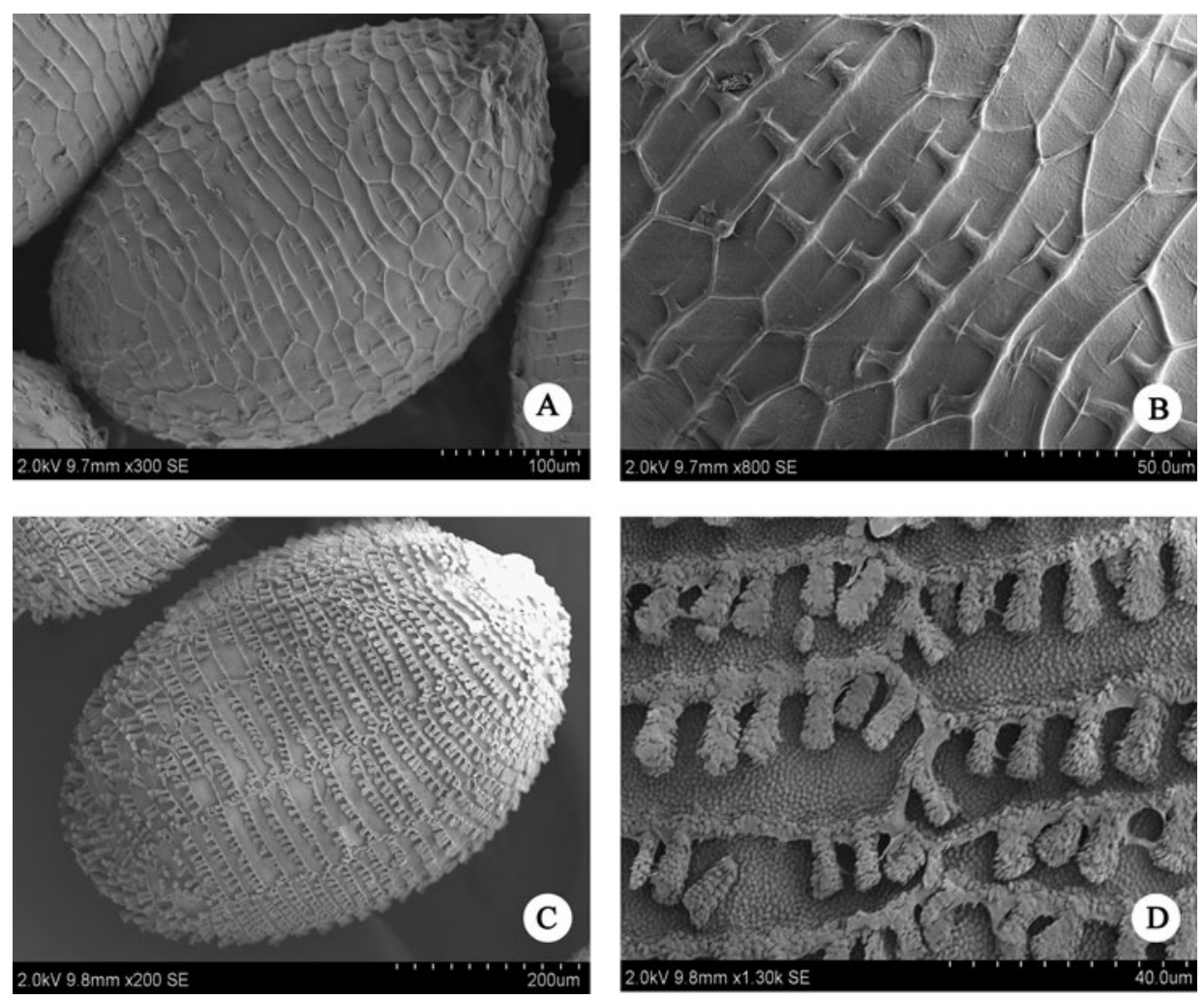

FIG. 2. SEM micrographs of the seed-coat of Eriocaulon malabaricum Pradeep \& Nampy and E. pykarense Nampy \& Manudev. A-B. E. malabaricum: A, entire seed; B, detail of seedcoat. C-D. E. pykarense: C, entire seed; D, detail of seed-coat.

shortly acuminate, hoary dorsally towards apex. Male flowers: Pedicels c. $0.4 \mathrm{~mm}$ long, glabrous. Sepals 3, connate; spathe obovate, c. $1.4 \times 0.75 \mathrm{~mm}$; lobes acute or obtuse, glabrous or occasionally hoary towards apex. Corolla tube c. $0.8 \mathrm{~mm}$ long. Petals 3 , minute, equal, ovate-obovate, glabrous, without a black gland. Stamens 6; filaments c. $0.25 \mathrm{~mm}$ long; anthers black. Female flowers: Pedicels 0. Sepals 3, free, dimorphic, oblanceolate, margins distally ciliate, acute at apex; laterals $1.2-1.5 \times 0.35-0.45 \mathrm{~mm}$, distinctly keeled, irregularly dentate at back; medial $1-1.2 \times 0.25-0.35 \mathrm{~mm}$, not distinctly keeled. Petals 3, equal, oblong-spathulate, $1.25-1.5 \times 0.1-0.15 \mathrm{~mm}$, obtuse, hoary towards apex, with a black gland. Ovary sessile, broadly ovate, $0.5 \times 0.4 \mathrm{~mm}$; style $0.4 \mathrm{~mm}$ long, 3-fid at apex. Seeds ovoid, seed-coat cells transversely elongated, aligned in vertical rows; appendages 1-3 from transverse radial cell walls, setiform, dilated at apex.

Distribution. Known only from Wayanad District in Kerala.

Habitat and ecology. Usually found growing along marshy areas.

Phenology. December-March. 
Etymology. The species is named after 'Malabar', one of the floristically rich regions in southern India, which includes the type locality, Kuppadi hills.

Taxonomic note. Eriocaulon malabaricum Pradeep \& Nampy is allied to E. sollyanum Royle but differs in having a cylindric receptacle, glabrous male petals without a black gland, dimorphic female sepals and 1-3 seed appendages arising from the transverse radial cell walls as opposed in E. sollyanum to a hemispherical or ovoid receptacle, hoary male petals with a black gland, monomorphic female sepals and 3-6 seed appendages arising from transverse radial cell walls. A more detailed morphological comparison of these two species is given in Table 1.

Additional specimens examined. INDIA. Kerala, Wayanad District, Sulthan Bathery, Kuppadi hills, 920 m, 10 ii 2010, in marshy areas, Sheba M. Joseph \& Santhosh Nampy 3011 (DEV); Muthanga, 800 m, Ratheesh Narayanan s.n. (CALI).

Eriocaulon pykarense Nampy \& Manudev, sp. nov. Figs 2C-D, 3.

Eriocauloni ansarii Pradeep \& Sunil affinis, a quo imprimis differt sepalis masculinis incanis, petalis masculinis glandulis, sepalis femineis ciliatis, seminum appendiculis 9-11 e parietibus transversalibus radialibus et 2 e parietibus verticalibus radialibus. - Type: India, Tamil Nadu, Nilgiris District, Pykara, 1420 m, on wet soil, 23 ii 2010, Santhosh Nampy 2757 (holo CALI; iso DEV, E).

Acaulescent herbs. Rootstock up to $5 \mathrm{~cm}$ long. Leaves acicular, $2-7 \times 0.2-0.6 \mathrm{~cm}$, glabrous. Peduncles 1-3(-7), 6-26 cm long, glabrous, striate; sheath up to $5 \mathrm{~cm}$ long, glabrous; limb entire, acute to acuminate at apex. Heads hemispherical, up to $5 \mathrm{~mm}$ across, black. Receptacle hemispherical, pilose. Involucral bracts narrowly to broadly obovate, $1.75-2 \times 1.3-1.5 \mathrm{~mm}$, spreading or reflexed, glabrous, rounded at apex. Floral bracts obovate, c. $2.5 \times 1.5 \mathrm{~mm}$, acuminate, hoary dorsally towards apex. Male flowers: Pedicels c. $0.6 \mathrm{~mm}$ long, glabrous. Sepals 3, connate; spathe

TA в LE 1. Comparison between Eriocaulon malabaricum and E. sollyanum

\begin{tabular}{|c|c|c|}
\hline Character & Eriocaulon malabaricum & Eriocaulon sollyanum \\
\hline Rootstock & Present & Absent \\
\hline Leaves & c. $4 \times 0.2 \mathrm{~cm}$ & c. $9 \times 0.3 \mathrm{~cm}$ \\
\hline Receptacle & Cylindric, pilose & $\begin{array}{l}\text { Hemispherical or ovoid, glabrous } \\
\text { or sparsely pilose }\end{array}$ \\
\hline Involucral bracts & $\begin{array}{l}\text { Obovate, acuminate } \\
\text { at apex }\end{array}$ & Oblong-spathulate, obtuse at apex \\
\hline Floral bracts & Obovate & Oblanceolate-cuneate \\
\hline Male flowers: Petals & $\begin{array}{l}\text { Glabrous, without a } \\
\text { black gland }\end{array}$ & $\begin{array}{l}\text { Glabrous or sparsely hoary, with } \\
\text { a black gland }\end{array}$ \\
\hline Female flowers: Sepals & $\begin{array}{l}\text { Dimorphic, keels } \\
\text { irregularly dentate }\end{array}$ & Monomorphic, keels entire \\
\hline Seed-coat appendages & $\begin{array}{l}\text { 1-3 from transverse } \\
\text { radial walls }\end{array}$ & 3-6 from transverse radial walls \\
\hline
\end{tabular}




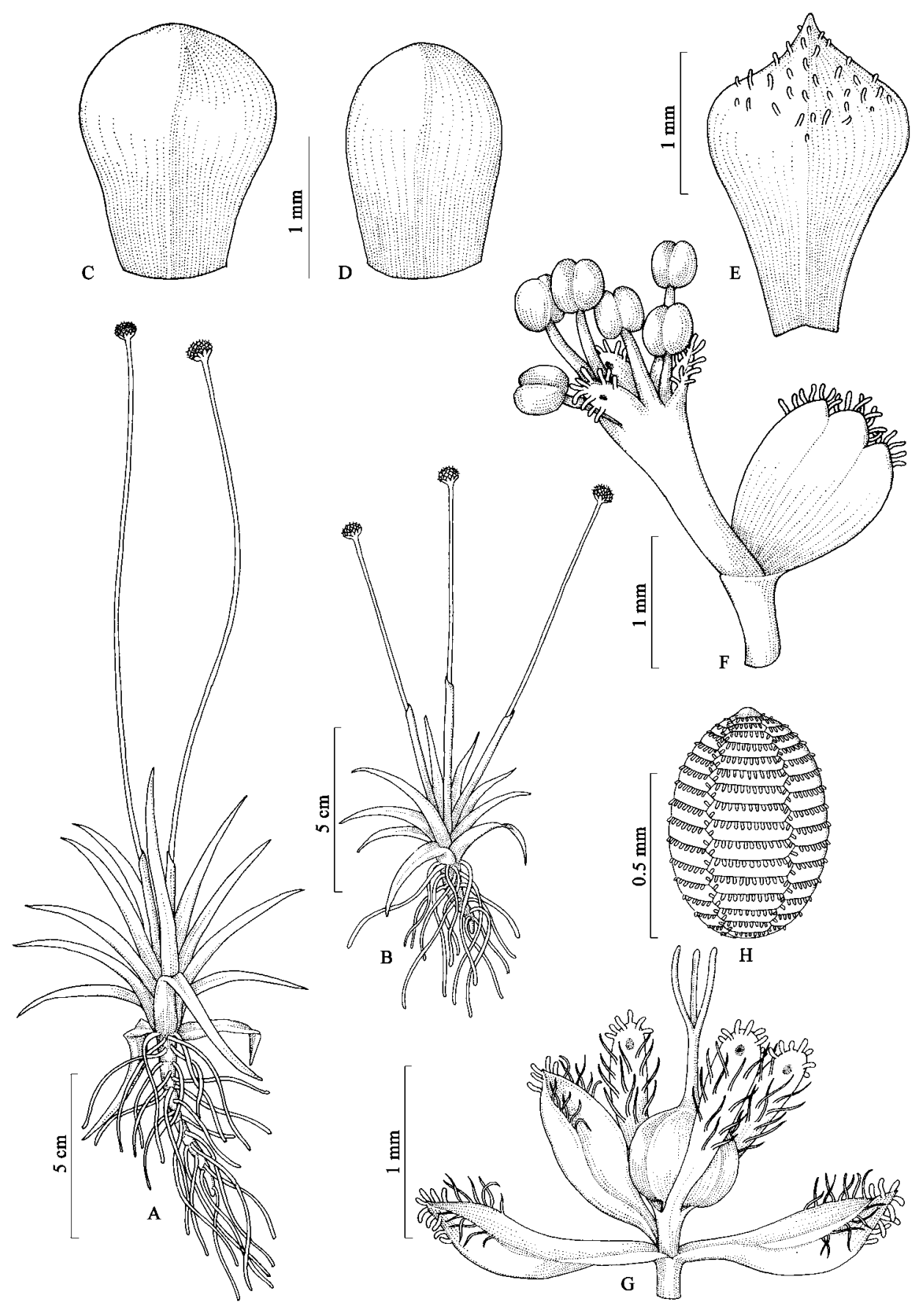

FIG. 3. Eriocaulon pykarense Nampy \& Manudev. A-B, habit; C-D, involucral bracts; E, floral bract; F, male flower; G, female flower; H, seed. Drawn by Manudev from the holotype. 
obovate, $1.2-1.4 \times 0.8-1 \mathrm{~mm}$; lobes acute or obtuse, hoary towards apex. Corolla tube 1.5-2 mm long. Petals 3, minute, equal, obovate, c. $0.5 \times 0.25 \mathrm{~mm}$, with a black gland, hoary towards apex. Stamens 6; filaments c. $0.75 \mathrm{~mm}$ long; anthers black. Female flowers: Pedicels $0.4 \mathrm{~mm}$ long. Sepals 3, free, black, oblanceolate, 1.75-2 $\times$ 0.6-0.75 mm, not distinctly keeled, margins ciliate, acute, hoary along keels and apex. Corolla tube c. $0.4 \mathrm{~mm}$ long. Petals 3, equal, spathulate, $1.5-1.75 \times 0.25$ $0.38 \mathrm{~mm}$, obtuse, pilose, hoary towards apex, with a black gland. Ovary sessile or subsessile, widely ovate, c. $0.9 \times 0.8 \mathrm{~mm}$; style $0.75 \mathrm{~mm}$ long; stigma $0.75 \mathrm{~mm}$ long, barbate. Seeds elliptic, seed-coat cells transversely elongated, aligned in vertical rows; appendages $9-11$ from transverse radial cell walls and 2 from vertical radial cell walls, setiform, truncate at apex.

Distribution. Known only from the type locality.

Habitat and ecology. Marshy places in grasslands.

Phenology. December-February.

Etymology. The species is named after the type locality Pykara, in southern India.

TA в Le 2. Comparison between Eriocaulon pykarense, E. ansarii and E. sollyanum

\begin{tabular}{|c|c|c|c|}
\hline Character & Eriocaulon pykarense & Eriocaulon ansarii & Eriocaulon sollyanum \\
\hline Rootstock & Present, up to $5 \mathrm{~cm}$ long & $\begin{array}{r}\text { Present, up to } \\
1.2 \mathrm{~cm} \text { long }\end{array}$ & Absent \\
\hline Leaves & Acicular, 2-7 cm long & $\begin{array}{l}\text { Acicular, up to } \\
1 \mathrm{~cm} \text { long }\end{array}$ & Linear, c. $9 \mathrm{~cm}$ long \\
\hline Floral bracts & $\begin{array}{l}\text { Hoary dorsally towards } \\
\text { apex }\end{array}$ & Not hoary & $\begin{array}{l}\text { Hoary dorsally towards } \\
\text { apex }\end{array}$ \\
\hline \multirow{4}{*}{$\begin{array}{l}\text { Male flowers: } \\
\text { - Sepals } \\
\text { - Corolla tube } \\
\text { - Petals }\end{array}$} & & & \\
\hline & Hoary towards apex & Not hoary & Hoary towards apex \\
\hline & $1.5-2 \mathrm{~mm}$ long & c. $0.5 \mathrm{~mm}$ long & $0.5-0.75 \mathrm{~mm}$ long \\
\hline & $\begin{array}{l}\text { Hoary towards apex, } \\
\text { with a black gland }\end{array}$ & $\begin{array}{l}\text { Not hoary, without } \\
\text { any black gland }\end{array}$ & $\begin{array}{l}\text { Glabrous or sparsely } \\
\text { hoary, with a black } \\
\text { gland }\end{array}$ \\
\hline \multirow{2}{*}{$\begin{array}{l}\text { Female flowers: } \\
\text { - Sepals }\end{array}$} & & & \\
\hline & $\begin{array}{l}\text { Not distinctly keeled, } \\
\text { hoary along back and } \\
\text { apex, margins ciliate }\end{array}$ & $\begin{array}{l}\text { Not keeled, not hoary, } \\
\text { margins not ciliate }\end{array}$ & $\begin{array}{l}\text { Keeled, hoary along } \\
\text { back, margins not } \\
\text { ciliate }\end{array}$ \\
\hline - Petals & $\begin{array}{l}\text { Spathulate, pilose, with } \\
\text { a black gland, hoary } \\
\text { towards apex }\end{array}$ & $\begin{array}{l}\text { Elliptic, pilose, with } \\
\text { or without black } \\
\text { gland, not hoary }\end{array}$ & $\begin{array}{c}\text { Spathulate, not pilose, } \\
\text { with a black gland, } \\
\text { hoary towards apex }\end{array}$ \\
\hline $\begin{array}{l}\text { Seed-coat } \\
\text { appendages }\end{array}$ & $\begin{array}{l}\text { 9-11 from transverse } \\
\text { radial walls, } 2 \text { from } \\
\text { vertical radial walls, } \\
\text { truncate at apex }\end{array}$ & $\begin{array}{l}3 \text { or } 4 \text { from transverse } \\
\text { radial walls, truncate } \\
\text { at apex }\end{array}$ & $\begin{array}{l}\text { 3-6 from transverse } \\
\text { radial walls, dilated } \\
\text { at apex }\end{array}$ \\
\hline
\end{tabular}


Taxonomic note. Eriocaulon pykarense Nampy \& Manudev is allied to E. ansarii Pradeep \& Sunil but differs in having hoary male sepals, glandular male petals, ciliate female sepals, 9-11 seed-coat appendages arising from transverse radial cell walls and 2 seed-coat appendages arising from vertical radial cell walls as opposed to glabrous male sepals, non-glandular male petals, glabrous female sepals and 3 or 4 seed-coat appendages arising from transverse radial cell walls in E. ansarii.

Ansari (pers. comm. dated 19 May 2010) assigned our material to E. trilobum Buch.-Ham. ex Körn. which is conspecific with E. sollyanum Royle. However, Eriocaulon pykarense differs from E. sollyanum in having c.5 cm long rootstock, acicular leaves, projecting male flowers, pilose female petals and 9-11 seed appendages arising from transverse radial cell walls and 2 from vertical radial cell walls as opposed to the absence of a rootstock, linear leaves, male flowers equalling the head, glabrous female petals and the number of seed appendages arising from transverse radial cell walls not exceeding 6 in E. sollyanum. A more detailed morphological comparison of these three species is given in Table 2.

\section{ACKNOWLEDGEMENTS}

We are grateful to Dr R. Ansari, an authority on Indian Eriocaulaceae, for his critical comments on our specimens. S.N. and M.K.M. are grateful to Dr C. J. Mani, Head, Department of Botany and Fr. Benny Sebastian, Principal, St Joseph's College, Kozhikode-8, for facilities; Mr Johny V.C. for helping with the collections; and Mrs Sheba M. Joseph for providing specimens for Eriocaulon malabaricum. S.N. is grateful to the University Grants Commission, Southwestern Circle, Bangalore for financial assistance.

\section{REFERENCES}

Ansari, R. \& BAlakrishnan, N. P. (2009). The family Eriocaulaceae in India. Revised edition. Dehra Dun: Bishen Singh Mahendra Pal Singh.

Mabberley, D. J. (2008). Mabberley's Plant-Book: A Portable Dictionary of Plants, Their Classification and Uses, 3rd edition. Cambridge: Cambridge University Press.

Shimpale, V. B., Bhagat, R. B., Deshmukh, R. B. \& Yadav, S. R. (2009). A new species of Eriocaulon (Eriocaulaceae) from Maharashtra, India. Rheedea 19: 47-49.

Yadav, S. R., Potdar, G. G., Anil Kumar, A., Otaghvari, M. \& Sonkar, A. (2008). Eriocaulon epedunculatum, a new species of Eriocaulaceae from Western Ghats of India. Kew Bull. 63: 503-505. 